\title{
Structure and Properties of Heavily B and P Co-doped Amorphous Silicon Quantum Dots Supporting Information
}

\author{
R. Turanský, ${ }^{\dagger}$ J. Brndiar, ${ }^{\dagger}$ A. Pershin, ${ }^{\ddagger}$ Á. Gali, ${ }^{* \dagger}$ H. Sugimoto, ${ }^{\uparrow}$ M. Fujii, ${ }^{*},{ }^{\top}$ and \\ I. Štich ${ }^{*, \dagger, \S, \|}$ \\ $\dagger$ Center for Comp. Mat. Science, Inst. of Physics, Slovak Acad. of Sciences, 84511 \\ Bratislava, Slovakia \\ $\ddagger$ Wigner Research Centre for Physics, Institute for Solid State Physics and Optics, P.O. \\ Box 49, H-1525 Budapest, Hungary \\ I Department of Electrical and Electronic Engineering, Graduate School of Engineering, \\ Kobe University, Japan \\ $\S$ Inst. of Informatics, Slovak Acad. of Sciences, 84507 Bratislava, Slovakia \\ ||Department of Natural Sciences, University of Ss. Cyril and Methodius, 91701 Trnava,
} Slovakia.

E-mail: gali.adam@wigner.hu; fujii@eedept.kobe-u.ac.jp; ivan.stich@savba.sk

\section{Nanoparticle preparation}

We now describe preparation of quantum dots (QDs) of different sizes and dopant concentrations focussing on one particular system, a small $\mathrm{B}$ - and P-codoped $\mathrm{Si}$ nanoparticle, $\mathrm{Si}_{134} \mathrm{~B}_{24} \mathrm{~B}_{14} \mathrm{H}_{52}$. Unless stated otherwise, qualitatively similar conclusion are valid also for the other NPs we prepared.

The general strategy is to start with a crystalline QD with a given dopant concentration and melt it to the point where all atoms, including the dopants, would exhibit non-negligible diffusion followed by a (rapid) quench. This is reminiscent of what was previously applied also to bulk liquid/amorphous elemental semiconductor systems. ${ }^{1,2}$ We have initially included also hydrogen termination to saturate surface dangling bonds. This last step turned out to be counterproductive as the H-termination rendered the outer shell rigid, created huge temperature gradients across the QD, which ultimately led to NPs with unrealistic atomic structure with very narrow, difficult to improve, electronic gaps. Therefore, before any thermal treatment the hydrogen termination was removed, $\mathrm{Si}_{137} \mathrm{~B}_{24} \mathrm{~B}_{14}$, Supporting Fig. S1a. The basic steps of our preparation procedure are described in more detail below. We note that the steps could be automated, if more QDs were to be prepared.

\section{Nanoparticle melting}

The temperature treatment started at $\mathrm{T}=$ $500 \mathrm{~K}$ by increasing the temperature in steps of $100 \mathrm{~K}$, Supporting Fig. S1b. The mean square displacement of atoms ${ }^{3}\left(R^{2}(t)\right)$ was monitored untill we observed diffusion of all three species. An example of $R^{2}(t)$ at $\mathrm{T}=$ 1500 K, Supporting Fig. S1e, shows a linear increase with time, indicating that the QD was indeed melted. Diffusion coefficients extracted from the mean squared displacements via Enstein's relation ${ }^{3}$ yield $D_{S i} \approx$ $0.6 \times 10^{-4} \mathrm{~cm}^{2} \mathrm{~s}^{-1}, \quad D_{B} \quad \approx 0.09 \times 10^{-4} \mathrm{~cm}^{2} \mathrm{~s}^{-1}$, and $D_{P} \approx 0.07 \times 10^{-4} \mathrm{~cm}^{2} \mathrm{~s}^{-1}$. The value of $0.6 \times 10^{-4} \mathrm{~cm}^{2} \mathrm{~s}^{-1}$, for $D_{S i}$ is of the same order 
of magnitude albeit smaller than that observed previously for bulk liquid silicon at $\mathrm{T}=1800 \mathrm{~K}$ $\left(2.2 \times 10^{-4} \mathrm{~cm}^{2} \mathrm{~s}^{-1}\right)$, which is consistent with the lower temperature, small finite QD, and presence of dopants.

The coordination number distribution, Supporting Fig. S1g, indicates the prevalent coordination number for Si of 4 , for our definition of a bonded atom see Sect. , which is smaller than 6.5 found in the bulk liquid at $\mathrm{T}=1800 \mathrm{~K} .{ }^{1}$ This is due to the large surface/bulk fraction which leaves many atoms on the surface with a lower coordination than that in the bulk. Contrary, atoms inside the QD are often overcoordinated (5-, 6-fold), leading to a rather broad distribution of Si coordination numbers. While boron forms three-center bonds ${ }^{4}$ and generally prefers higher coordination the $\mathrm{B}$ atoms reside preferentially in the outer cluster shell, leading to a coordination number distribution very similar to that of Si, Fig. 1g. Note that the situation is different in absence of phosphorus, in which case boron atoms protrude to the QD core and the coordination of $\mathrm{B}$ atoms increases to 5 as shown in Figure 2 of the main text. Phosphorus prefers a 3 -fold coordination which is also reflected by the coordination number distribution in the liquid, closely followed by 2 -fold one due to the thermal motion.

Subsequently the melt was in steps of $100 \mathrm{~K}$ cooled down to 400K, Supporting Fig. S1b. As signalled by mean square displacement, atoms stopped diffusing at around 700K, see Supporting Fig. S1f. The final distribution of coordination numbers in the quenched liquid, Supporting Fig. S1h, shows that all coordination number distributions narrowed significantly and became strongly peaked at 4 for $\mathrm{Si}$ and $\mathrm{B}$, and 3 for P.

\section{Saturation of dangling bonds and elimination of gap states}

The as-quenched NPs, Supporting Fig. S1c, were essentially metallic. This can be seen by inspecting the distribution of Kohn-Sham (KS) single particle (PBE) energies, see Supporting Fig. S2.

The gap states have two different origins, the undercoordinated atoms residing on the QD surface, and the overcoordinated atoms in the QD core due to the rapid quench. The electronic gap was opened up by three different means: 1) saturation of dangling bonds at the QD surface by hydrogen atoms, 2) local reoptimization of the atomic structure to remove overcoordinated atoms by short-time molecular dynamics runs at temperatures allowing only for very sluggish diffution (700-1000K) followed by local reoptimization to remove the thermal disorder, 3) by eliminating overcoordinated atoms (as in grand canonical ensemble) followed by local structure reoptimization. The steps 1)-3) were guided by monitoring the few electronic states around the HOMO and LUMO levels while keeping the system in singlet state. As can be seen in Supporting Fig. S2, most gap states were eliminated by hydrogenation. Step 2) was more controversial, as MD structural reoptimization often led to structures which healed one coordination defect but created a new one(s) elsewhere. More efficient means of elimination of critical overcoordination defects turn out step 3), removal of overcoordinated atoms. Supporting Fig. S2 also shows that elimination of a coordination defect (in presence of other coordination defects) does not always lead to HOMO-LUMO gap increase. This is because another, lower in energy gap state(s), no longer kept by the orthogonality constraint(s) to the electronic gap state higher in energy, can now propagate to the gap. Hence, a number of correlated steps have to be made to clear up/open up the QD electronic gap. 17 such steps have been made in this particular system, not counting those not deemed successful in improving the structure. Such steps have been scrapped and a different optimization route sought. The final QD obtained by this procedure, $\mathrm{Si}_{134} \mathrm{~B}_{24} \mathrm{P}_{14} \mathrm{H}_{52}$, is depicted in Supporting Fig. S1d. We note that application of steps 1) - 3) led to gap opening from 0 to $\approx 0.8 \mathrm{eV}$ at $\mathrm{PBE}$ level ${ }^{5,6}$ or $\approx 1.6 \mathrm{eV}$ at B3LYP level, ${ }^{7,8}$ and $\approx 1.3$ at HSE06 level. ${ }^{9}$ 

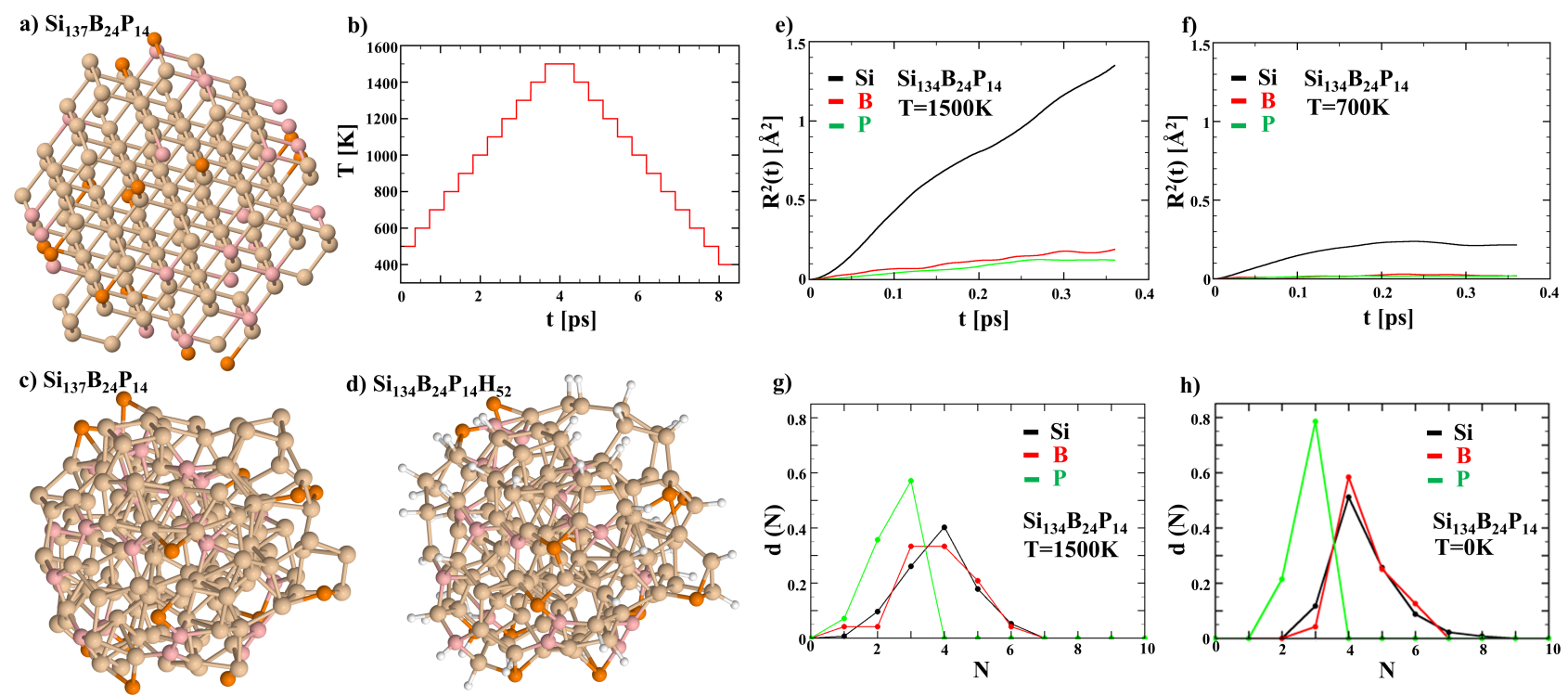

Supporting Fig. S 1: Preparation of a B-P-codoped Si nanoparticle. a Initial crystalline Si nanoparticle with $\mathrm{B}$ and $\mathrm{P}$ dopants without hydrogen termination. $\mathbf{b}$ Schedule of thermal treatment. $\mathbf{c}$ Ball and stick model of the as quenched B-P-codoped Si nanoparticle without hydrogen termination. d Final structure after adding hydrogen termination and opening the band gap, see Supporting Fig. S2. e-f Mean square displacements of the Si, B, and P atoms at $\mathrm{T}=1500 \mathrm{~K}$ and $\mathrm{T}=700 \mathrm{~K}$, respectively. g-h Distribution of coordination numbers, for definition see Supporting Fig. S3, in the nanoparticle at $\mathrm{T}=1500 \mathrm{~K}$ and $\mathrm{T}=0 \mathrm{~K}$ (as quenched), respectively

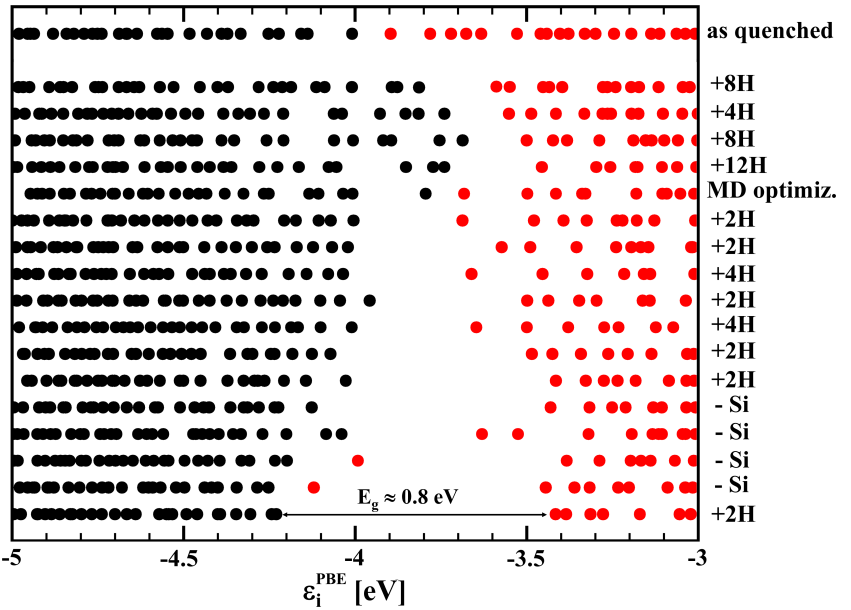

Supporting Fig. S 2: DFT (PBE) singleparticle energies (black occupied states, red unoccupied states) around the Fermi energy at different stages of nanoparticle preparation, the nanoparticle state/modification is indicated on the right. This figure only shows steps deemed successful in improving the QD structure.

\section{Characterisation of bonds in the nanoparticle}

In an amorphous system as well as in liquid there is a continuous distribution of bond types ranging from very strong (compressed bonds) to very weak extended/broken bonds. Hence, if a neighbor is to be identified based on bonding, a suitable bond cut-off has to be found for all (6) combination of atom pairs. An example for three different pairs including the $\mathrm{Si}$ atom are shown in Supporting Fig. S3 featuring always one strong and one weak bond. Based on such an analysis of bonding the following cutoffs were selected:

$\mathrm{R}_{\mathrm{Si}-\mathrm{Si}}=2.75 \AA$,

$\mathrm{R}_{\mathrm{Si}-\mathrm{P}}=2.65 \AA$,

$\mathrm{R}_{\mathrm{Si}-\mathrm{B}}=2.45 \AA$,

$\mathrm{R}_{\mathrm{B}-\mathrm{B}}=2.00 \AA$,

$\mathrm{R}_{\mathrm{P}-\mathrm{P}}=2.50 \AA$,

$\mathrm{R}_{\mathrm{B}-\mathrm{P}}=2.00 \AA$,

$\mathrm{R}_{\mathrm{Si}-\mathrm{H}}=1.80 \AA$,

$\mathrm{R}_{\mathrm{B}-\mathrm{H}}=1.70 \AA$,

$\mathrm{R}_{\mathrm{P}-\mathrm{H}}=1.60 \AA$,

$\mathrm{R}_{\mathrm{H}-\mathrm{H}}=1.80 \AA$. 

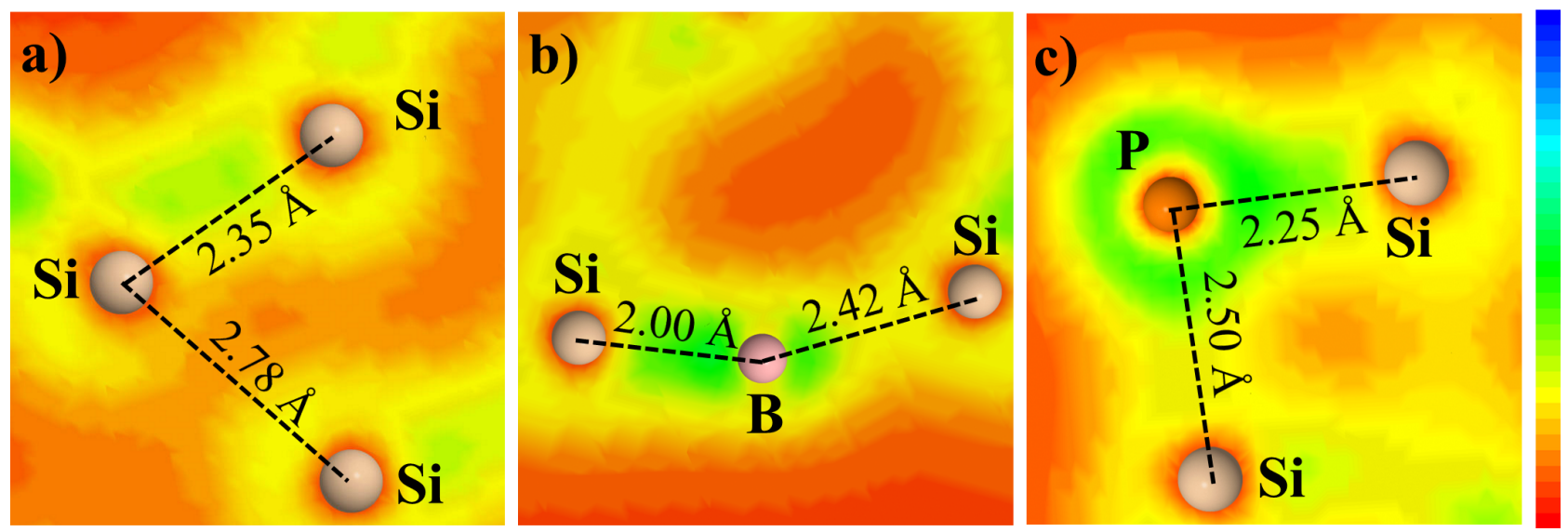

Supporting Fig. S 3: Electronic (pseudo) charge densities highlighting the concept of strong and weak/no bond concept for an Si-Si (panel a), Si-B (panel b), and P-Si (panel c) atom bond.

We note that these cut-offs are generally somewhat smaller than those customarily used by computer codes such as VASP. ${ }^{10}$

\section{Nanoparticle electronic struc- ture: Projected densities of states}

In Supporting Fig. S4 we show the densities of electronic states calculated in the PBE treatment for the $\mathrm{Si}_{286} \mathrm{~B}_{61} \mathrm{P}_{36} \mathrm{H}_{59}$ QD projected onto $s$ - and $p$-states of $\mathrm{Si}, \mathrm{B}, \mathrm{P}$, and $\mathrm{H}$ ( $s$-states only) atomic species. A rough division point between the $s$ - and $p$-states is $\approx-5 \mathrm{eV}$. The dopants, es-

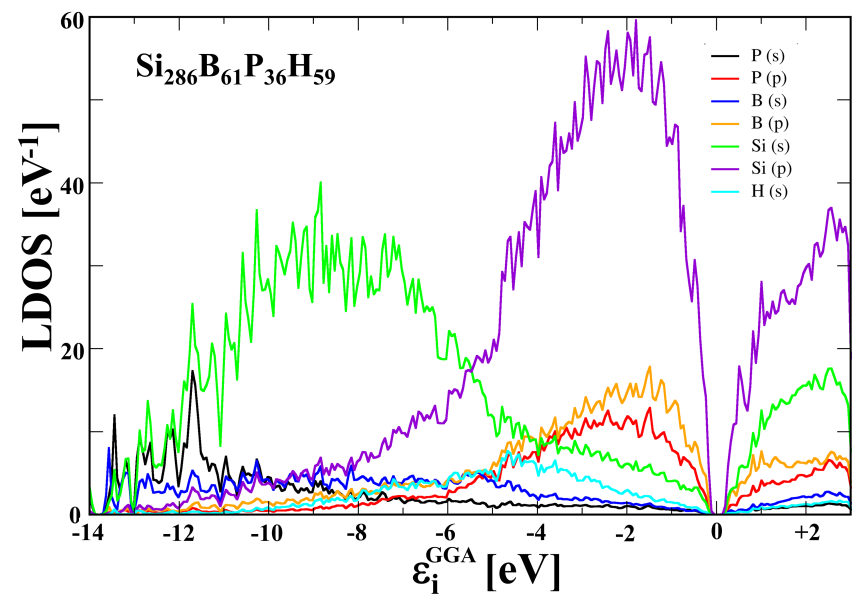

Supporting Fig. S 4: Densities of electronic states (PBE XC functional) in the $\mathrm{Si}_{286} \mathrm{~B}_{61} \mathrm{P}_{36} \mathrm{H}_{59}$ QD projected onto different atomic species and orbital moments.

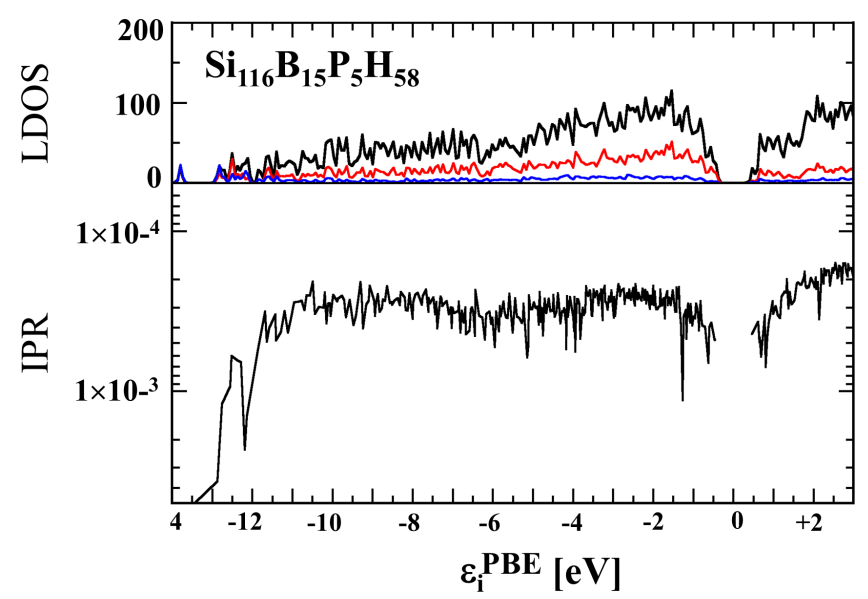

Supporting Fig. S 5: Projected electronic densities of states (upper panels, total black, boron red (multiplied by 5 for clarity), phosphorus blue (multiplied by 5 for clarity) and inverse participation ratios (lower panels) for the $\mathrm{Si}_{116} \mathrm{~B}_{15} \mathrm{P}_{56} \mathrm{H}_{58}$ QD. 

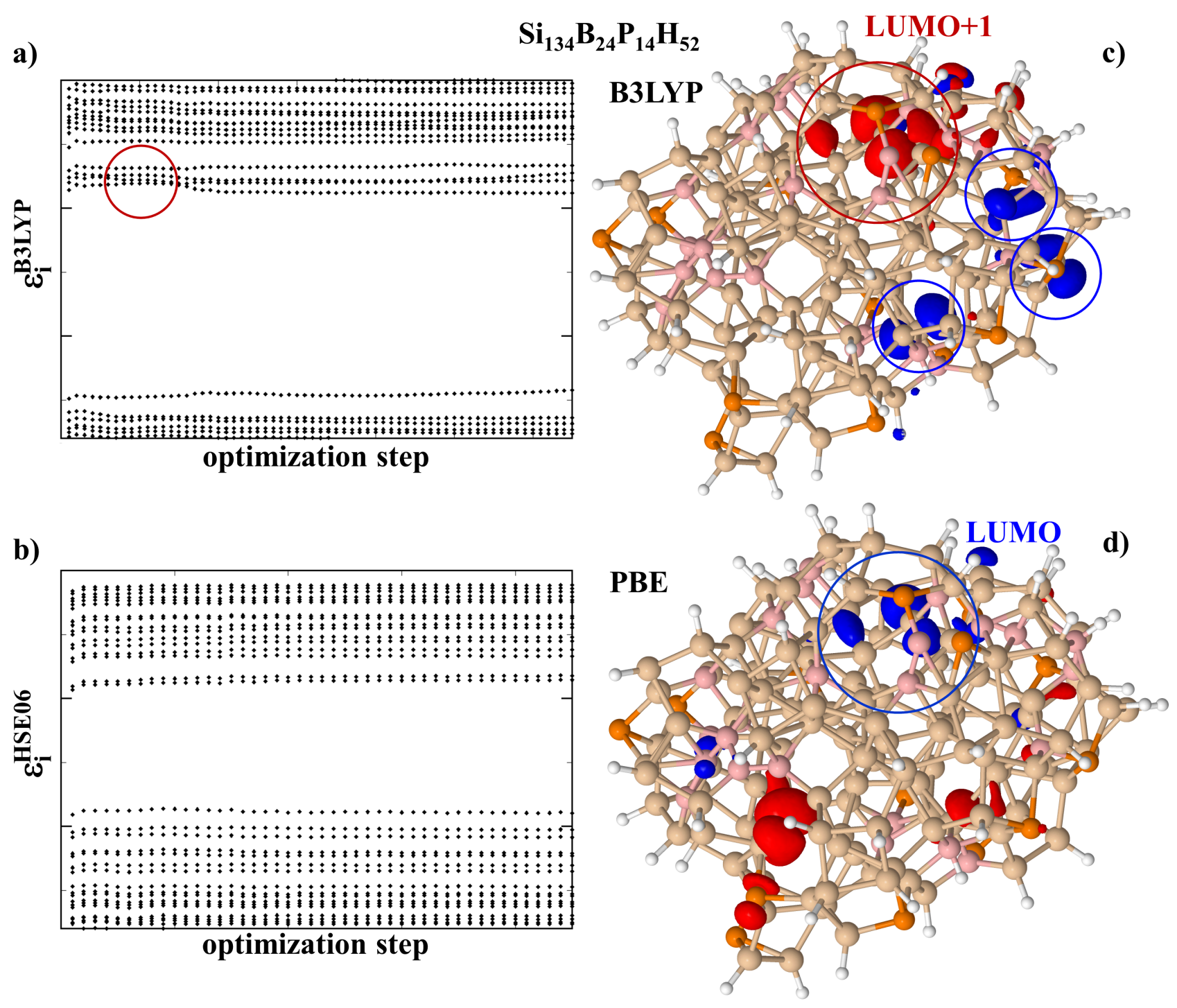

Supporting Fig. S 6: Convergence of electronic gaps and single-particle K-S orbitals around the band gap. Convergence of a B3LYP hybrid, b HSE06 hybrid K-S single particle energies. The red circle in a indicates an (avoided) level crossing. c Electronic charge densities corresponding to LUMO (blue) and LUMO+1 (red) K-S orbitals in B3LYP treatment and $\mathbf{d}$ ) in PBE treatmentat. The circles depict the orbital transformation upon structural relaxation. 


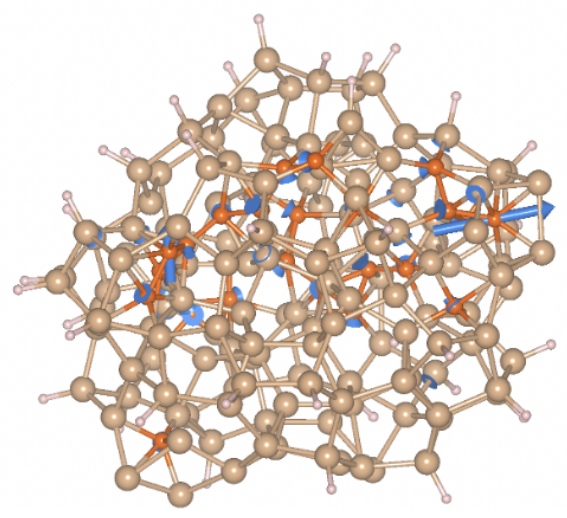

$694 \mathrm{~cm}^{-1}$

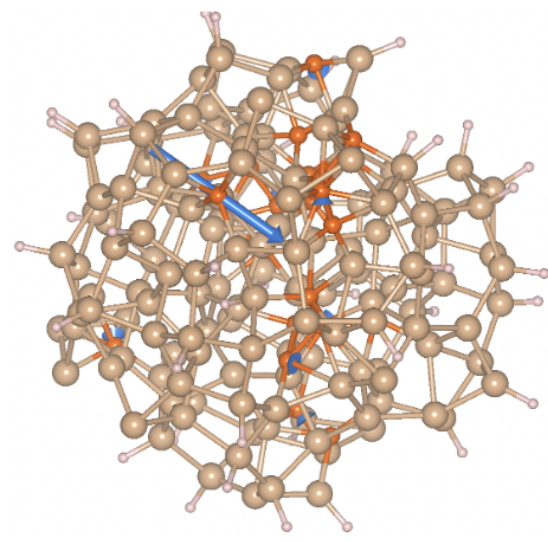

$812 \mathrm{~cm}^{-1}$

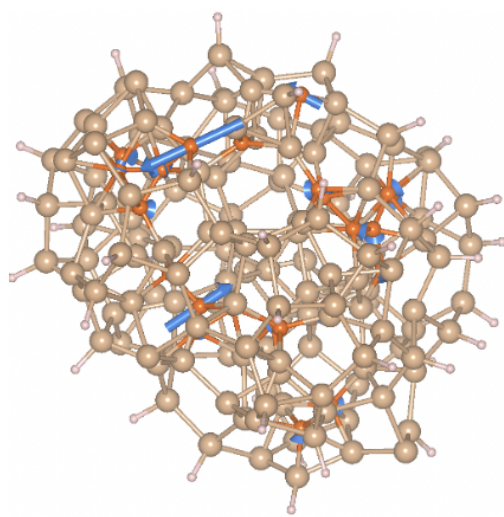

$860 \mathrm{~cm}^{-1}$

Supporting Fig. S 7: Several representative modes of the indicated frequencies, contributing to an enhanced activity in the Raman spectrum of $\mathrm{Si}_{147} \mathrm{~B}_{23} \mathrm{H}_{41}$. The magnitude of the vectors shows the participation of respective atoms. Colour code: green is for boron atoms, blue is for silicon, and white is for the hydrogens.

pecially phosphorus, have very low $s$-states below $\approx-10 \mathrm{eV}$. The $p$-states in all three atomic species ( $\mathrm{Si}, \mathrm{B}, \mathrm{P})$ do largely overlap in energy, and hence tend to hybridize, which is partially true even for the $\mathrm{P}$ lone pair electrons. The results for the other NPs look qualitatively similar. Supporting Fig. S 4 is especially useful in connection with analysis of IPRs in Fig. 3 of the main text.

\section{Additional electronic densi- ties of states and IPRs}

In Supporting Fig. S5 we show the projected densities of electronic states and the corresponding IPR for the other small $\mathrm{B}$ and $\mathrm{P}$ codoped QD, the $\mathrm{Si}_{116} \mathrm{~B}_{15} \mathrm{P}_{5} \mathrm{H}_{58}$. These results are qualitatively very similar to those for the $\mathrm{Si}_{134} \mathrm{~B}_{24} \mathrm{P}_{14} \mathrm{H}_{52}$ QD shown in Figure of the main text.

\section{Nanoparticle electronic gaps: GGA versus hybrid func- tionals}

In Tab. I of the main text we compile the fundamental electronic gaps of our model QDs calculated by $\mathrm{PBE}^{5,6}, \mathrm{HSE}^{9}{ }^{9}$, and $\mathrm{B} 3 \mathrm{LYP}^{7,8}$ exchange correlation functionals. The hybrid functionals were applied both perturbatively on the PBE geometries as well as self-consistently on the geometries relaxed by hybrid functionals. The differences between the perturbative and fully consistent approach are minor, 0.05$0.1 \mathrm{eV}$. In Supporting Fig. S6 we show the convergence of the gap and of the few unoccupied/occupied orbitals as a function of structural optimization step for our $\mathrm{Si}_{134} \mathrm{~B}_{24} \mathrm{P}_{14} \mathrm{H}_{52}$ QD. While no qualitative changes occur in the HSE06 treatment (see panel b), due to much stronger structural relaxation in the B3LYP treatment, (avoided) level crossings do occur (panel a). The changes at the orbital level are depicted in panels $\mathbf{c}$ and $\mathbf{d}$ which show that the PBE LUMO orbital becomes after relaxation the LUMO+1 orbital, whereas a new LUMO orbital arose by modification of the coordination of three Si atoms which became 3-fold coordinated in B3LYP relaxation (blue circles) while nominally 4-fold coordinated in the PBE treatment (panels $\mathbf{c}$ and $\mathbf{d}$ ). We have identified more such processes resulting from relaxing the structures to the B3LYP minima (not shown). These findings suggest that a more reliable B3LYP value of the electronic gap is obtained in the perturbative treatment. Such a conclusion is corroborated also by the hybrid values of the electronic gap due to selfconsistent treatment which is slightly opening 
the HSE06 gap while both reduction and increase of the B3LYP gap may occur, depending on the nature of the structural relaxation.

\section{Nanoparticle vibrational properties: Raman spectra}

Supporting Fig. S7 shows examples of representative modes contributing to enhanced activity in the Raman spectrum of $\mathrm{Si}_{147} \mathrm{~B}_{23} \mathrm{H}_{41}$.

\section{References}

(1) Štich, I.; Car, R.; Parrinello, M. Structural, Bonding, Dynamical, and Electronic Properties of Liquid Silicon: An Ab Initio Molecular Dynamics Study. Phys. Rev. B 1991, 44, 4262 .

(2) Štich, I.; Car, R.; Parrinello, M. Amorphous Silicon from Ab Initio Molecular Dynamics: Preparation, Structure, and Properties. Phys. Rev. B 1991, 44, 11092.

(3) Hansen, J. P.; McDonald, I. Theory of Simple Liquids; Academic: New York, 1976.

(4) Mayer, I. Bond orders in three-centre bonds: an analytical investigation into the electronic structure of diborane and the three-centre four-electron bonds of hypervalent sulphur. J. Mol. Struct. 1989, 186, $43-52$.

(5) Perdew, J. P.; Burke, K.; Ernzerhof, M. Generalized Gradient Approximation Made Simple. Phys. Rev. Lett. 1996, $77,3865$.

(6) Perdew, J. P.; Burke, K.; Ernzerhof, M. ERRATA: Generalized Gradient Approximation Made Simple. Phys. Rev. Lett. 1997, 78, 1396.

(7) Becke, A. D. Density functional thermochemistry. III. The role of exact exchange. J. Chem. Phys. 1993, 98, 5648-5652.
(8) Stephens, P. J.; Delvin, F. J.; Chabalowski, C. F.; Frisch, M. J. Ab Initio Calculation of Vibrational Absorption and Circular Dichroism Spectra Using Density Functional Force Fields. J. Phys. Chem. 1994, 98, $11623-11627$.

(9) Krukau, A. V.; Vydrov, O. A.; Izmaylov, A. F.; Scuseria, G. E. Influence of the exchange screening parameter on the performance of screened hybrid functionals. J. Chem. Phys. 2006, 125, 224106.

(10) Kresse, G.; Furthmüller, J. Efficiency of ab-initio total energy calculations for metals and semiconductors using a plane-wave basis set. Comput. Mater. Sci. 1996, 6, 15. 